\title{
Body dissatisfaction and pathogenic weight loss behaviors among Iranian women with different level of physical activity
}

\author{
Donya Nemati*, Farhad Rahmani-nia, Hamid Mohebbi, \\ Department of Physical Education and Sport Sciences, Rasht, Guilan, Iran \\ E-mail: Donya.nmt@gmail.com
}

\begin{abstract}
Body dissatisfaction is becoming increasingly pervasive in the developing non-Western countries such as Iran; however, there exists a lack of research investigating the factors associated with these problems. The current study examined body dissatisfaction, social pressure, and weight loss behaviors and the relationships between these variables. Our data collected from 711 women; elite athletes (non-lean sport) $(\mathrm{N}=211)$, nonelite athletes $(\mathrm{N}=93)$ and non-athletes $(\mathrm{N}=407)$ in Iran by employing a Photographic Figure Rating Scale (PFRS) and a questionnaire of pathogenic weight loss behaviors. Non-athletes reported greater body dissatisfaction, social pressure to change weight and pathogenic weight loss behaviors than the other groups. Body dissatisfaction was predicted by pressure from family and friends to change weight and followed by BMI in 3 groups. Body dissatisfaction was also positively correlated with pathogenic weight loss behaviors. Our results showed that non-athletes women experience greater body dissatisfaction, social pressure than elite athletes and non-elite athletes.
\end{abstract}

Keywords: body dissatisfaction, Iranian women, elite athletes, non-elite athletes, weight loss behaviors. 


\section{INTRODUCTION}

The models of beauty would impose a lean body shape without considering both healthrelated aspects and the diverse physical features of different societies [1]. The development of this unrealistic beauty standards increases body image dissatisfaction referring to a discrepancy between perceived and actual weight status among women [2]. In fact, women are more vulnerable to pressure of society and culture for aesthetic standards [3]. They are influenced by various sources of social pressure including friends, family, and media [4]. Evidence shows that perceived pressure from family and friends are more disturbing than media because family and friends are closest people around a person [5]. For example comments from family and friends regarding shape, weight, and eating have been shown to be an effective element on the prevalence of body dissatisfaction [6]. Hence, negative comments from parents about body shape have been found to cause body dissatisfaction [6]. A study of middle school girls indicated that after controlling for BMI, weight-related teasing by parents was the most significant predictor of body dissatisfaction, thin-ideal internalization, and restrictive eating behaviors [7]. Another study showed that among girls and boys, parental teasing causes body dissatisfaction and disordered eating [8]. Similarly, Ricciardelli \& McCabe [9] found out that social pressure was the strongest indicator of body change strategies among both genders. Besides, these risk factors lead an increasing number of women that undergo pathogenic weight loss behaviors such as excessive exercises, laxatives, diuretics, and diet pills to achieve unrealistic body shape [1]. These precursory behaviors of eating disorders are called a partial syndrome of eating disorder [1]. Detection of the partial syndrome of eating disorder is so important to prevent the unhealthy consequences [1]. 
By considering the fact that women have a higher tendency to show their beauty and attract attention, female athletes may also experience additional sports related pressure to achieve a certain ideal athletic body shape and body size as well [10]. Without any doubt the psychological benefits associated with exercise and sports activity are well known [11]. However, researchers suggest that exercise may result in adverse consequences in some conditions too. For instance, elite athletes or individuals with a high level of physical activity exhibit more eating pathology and disordered eating than non-athletes and recreational athletes or people who are exercising at moderate levels [12, 13]. Some studies have investigated that athletes are more at risk of disordered eating and body image dissatisfaction than non-athletes $[10,14]$. However, it is reported that athletes have a lower body dissatisfaction than non-athletes $[15,16,17]$.

Although in the last decade researchers have focused on Western women [18], Recent studies have shown an increase in body dissatisfaction and disordered eating in nonWestern countries too, like Malaysia [19] and China [20] to name a few. In other words, it is believed that the culture [21] and cover-and-dress preferences [13] are a crucial factor in the prevalence of body dissatisfaction. Thus it seems that study of body dissatisfaction in a society with the least possible approach to the Western culture and different cover-anddress preferences such as Iran can successfully extend the literature in this regard. Moreover, there have been very few studies exploring the prevalence of pathogenic weight loss behaviors and body dissatisfaction in Iran. In particular, to our knowledge, there has been no report on body dissatisfaction, social pressure, and pathogenic weight loss behaviors among Iranian athletes mainly female elite athletes. The current study aimed to extend the literature in this regard, in order to examine social pressure, body dissatisfaction 
and pathogenic weight loss behaviors among Iranian female athletes and non-athletes by employing the standard methods in different levels of competitions.

\section{MATERIALS AND METHOD}

Data for the present study were collected from 711 female volunteers between the aged of 18-30 years old in Iran. They were recruited by posting announcements at various locations in the dormitory to collect non-elite athletes and non-athletes samples. Also, we sent an invitation to the coaches of national sports team to ask their team to participate in our research as well. All study protocols were approved by the Guilan university research ethics review board before the initiation. Afterward, the non-athlete volunteers were recruited from the campus dormitory at the Guilan University and non-elite athletes were recruited from their teams at the University of Guilan as well.

To include all possible female elite athletes, we checked the timetables of the elite athletes in the campus in different fields of sports in Tehran, (the capital of Iran) and arranged for a visit. We directly contacted the athletes and obtained permission from their coaches and the varsity teams for elite and non-elite athletes, respectively, then in the interview a package of the questionnaires was administered to the participants by the researcher Donya Nemati. The package consisted of a cover letter, a consent form, a demographic information form, Photographic Figure Rating Scale and a questionnaire about the Pathogenic weight loss behaviors. Also, weight and height of all participants were measured by the researcher at the end of the interview. All participants volunteered anonymously before completing the questionnaires. 
Participants were divided into three groups: The first Group, 407 university students from a large public university of Guilan who did not have any regular physical activity, categorized as non-athletes, Second Group included 93 university students who participated in 5 sports events (volleyball, basketball, football, badminton, and handball) as a member of the varsity sports team, categorized as non-elite athletes. Since there are a limited number of Iranian female elite athletes at the international level, in line with the standards_observed in Iran, an elite athlete was defined as an athlete who competed in national sports teams [22]. Then the third group involved 211 female elite athletes who participated in 5 sports teams (volleyball, basketball, track and field, canoe polo, and rugby). In the current study, all the sports events that mentioned above for elite athletes were considered as non-lean sports [23]. Moreover, track and field events also considered as non-lean sports because none of the elite athletes in this field participated in a longdistance running competition [23, 24].

\section{Measurements}

\section{Demographics}

All participants provided their demographic details consisting of age, marital status, lifetime sporting activity, educational level.

\section{Body dissatisfaction and social pressure}

A Photographic Figure Rating Scale (PFRS) was used to appraise body dissatisfaction based on perceived and ideal body images, which have been shown to have a good testretest reliability and a high construct validity [25]. The PFRS consists of 10 photographic 
images of real women wearing light gray leotards and leggings that range from emaciated (low BMI) to obese (high BMI). The set of images were classified into five subcategories as follow: emaciated ( $<15 \mathrm{~kg} / \mathrm{m} 2)$, underweight $(15-18.5 \mathrm{~kg} / \mathrm{m} 2)$, normal (18.5-24.9 $\mathrm{kg} / \mathrm{m} 2)$, overweight (25.0-29.9 kg/m2), and obese (>30 kg/m2).

Participants were asked to select one image that looked most like their body (perceived), one image that they would most like to look like (desired) and one image which they believed their friends and family would most want them to have (friend and family desired). After measuring their weight and height, we calculated their actual BMI. The difference between perceived BMI and desired BMI was considered as body dissatisfaction. The difference between perceived BMI and what friends or family desired were regarded as social pressure. Although People are influenced by various sources of social pressure including friends, family and media, it is shown that perceived pressure from friends and family are more disturbing since they are closest of all [26, 27, 28]. As described before, perceived pressure from family and friends have more influence than media on a person [5], therefore, in this study, the social pressure from family and friends to change weight were only used to measure the perceived pressure from society.

\section{Pathogenic weight loss behaviors}

In the current study weight loss behaviors were categorized into three methods. The first category defines as unsafe (extreme) methods, includes a self-reported use of diet pills, laxatives, diuretics, and vomiting within the last two months as a method of losing weight. The second method refers to potentially unsafe self-reported dieting and fasting to lose weight in the last two month and finally the third methods which consider as a potentially 
safe method, is exercising or participation in any physical activity to lose weight in the last two month [2]

\section{Body mass index (BMI)}

Measures of height and weight were collected by using standardized protocols. BMI was defined as weight in kilograms divided by height in meters squared. BMI was categorized into four groups based on standard classifications which widely were used in studies on Iranian women $[29,30]$ : underweight $(\mathrm{BMI}<18.5)$, normal weight (BMI 18.524.9); overweight (BMI 25.0-29.9), and obese (BMI> 30.0).

\section{Statistical analysis}

Statistical analysis was calculated using the statistical package SPSS [31] version 16.0. A one-way analysis of variance test (ANOVA) [32] with a post-hoc Tukey adjustment was applied to examine differences among the groups. In addition, Pearson's correlation coefficient [33] was used in order to explain the relationship between variables. The level of significance was taken to be 0.05 for all the statistical analyses.

\section{Result}

Statistical results of demographic variables are presented based on percentages, scale means, and standard deviations for all the three subject groups. The age average of all participants was $21.06(S D=2.15)$, the mean of BMI was $21.80(S D=2.90)$ which is within the normal age range (20-25). 90.8\% were single and $71.7 \%$ were undergraduate students. 


\begin{tabular}{|c|c|c|c|c|c|c|c|}
\hline & \multicolumn{2}{|c|}{ Demographic $^{a}$} & \multicolumn{3}{|c|}{ Sporting activity ${ }^{\mathrm{b}}$} & \multicolumn{2}{|c|}{ PFRS $^{\mathrm{a}}$} \\
\hline & $\begin{array}{l}\text { Age } \\
\text { (SD) }\end{array}$ & $\begin{array}{c}\text { BMI } \\
\left(\mathrm{kg} / \mathrm{m}^{2}\right)\end{array}$ & Years & $\begin{array}{c}\text { Days per } \\
\text { Week }\end{array}$ & $\begin{array}{c}\text { Hours per } \\
\text { Session }\end{array}$ & $\begin{array}{c}\text { Body } \\
\text { Dissatisfaction }\end{array}$ & $\begin{array}{c}\text { Social } \\
\text { Pressure }\end{array}$ \\
\hline $\begin{array}{l}\text { Elite athletes } \\
\qquad(\mathrm{n}=211)\end{array}$ & $\begin{array}{l}19.73 \\
(2.12)\end{array}$ & $21.52(2.96)$ & $3.61(0.82)$ & $2.67(0.83)$ & $2.32(0.56)$ & $0.83(1.00)$ & $0.84(0.97)$ \\
\hline $\begin{array}{c}\text { Non-elite } \\
\text { athletes }(n=93)\end{array}$ & $\begin{array}{l}22.21 \\
(2.02)\end{array}$ & 21.05 (1.77) & $2.00(0.55)$ & $2.06(1.01)$ & $2.27(0.85)$ & $1.10(1.15)$ & $1.00(1.09)$ \\
\hline $\begin{array}{c}\text { Non-athletes } \\
(n=407)\end{array}$ & $\begin{array}{l}21.48 \\
(1.86)\end{array}$ & $22.10(3.11)$ & $1.18(0.55)$ & $1.15(0.36)$ & $1.52(0.53)$ & $1.50(1.21)$ & $1.47(1.11)$ \\
\hline $\mathrm{F}^{\mathrm{C}}$ & $72.15 * *$ & $6.06^{* *}$ & $388.32 * *$ & $88.15^{* *}$ & $42.57 * *$ & $30.17 * *$ & $24.50 * *$ \\
\hline
\end{tabular}

Table 1: Means, standard deviations, and between-group differences for participant demographics, sporting activity, PFRS responses ${ }^{\mathrm{a}} \mathrm{df}=(2,708),{ }^{\mathrm{b}} \mathrm{df}=(2,370),{ }^{\mathrm{c} A l l} \mathrm{~F}$-values were significant ${ }^{*} \mathrm{p}<0.001$. BMI $=$ Body Mass Index.

PFRS $=$ Photographic Figure Rating Scale

As expected, there are significant differences between-group in age and BMI (Table 1).

The post-hoc Tukey HSD test showed that the non-athletes have a significantly higher BMI than both the elite and the non-elite athletes $(\mathrm{p}<0.05)$, whereas the athlete's groups, are not significantly different from each other $(p>0.05)$. However, the elite athletes are youngest in compare to other groups. Table 1 also shows that there are significant differences between elite athletes, non-elite athletes, and non-athletes in the level of sporting activity, as expected, elite athletes reported more regular sporting activity than the other groups ( $\mathrm{p}<0.001)$. The differences between-group in body dissatisfaction and social pressure is significant too (Table 1).

The post-hoc Tukey test showed that the non-athletes have a significantly higher body dissatisfaction and social pressure than the other groups ( $\mathrm{p}<0.05)$, whereas the athlete's 
groups, are not significantly different from each other in body dissatisfaction and social pressure variables $(\mathrm{p}>0.001)$.

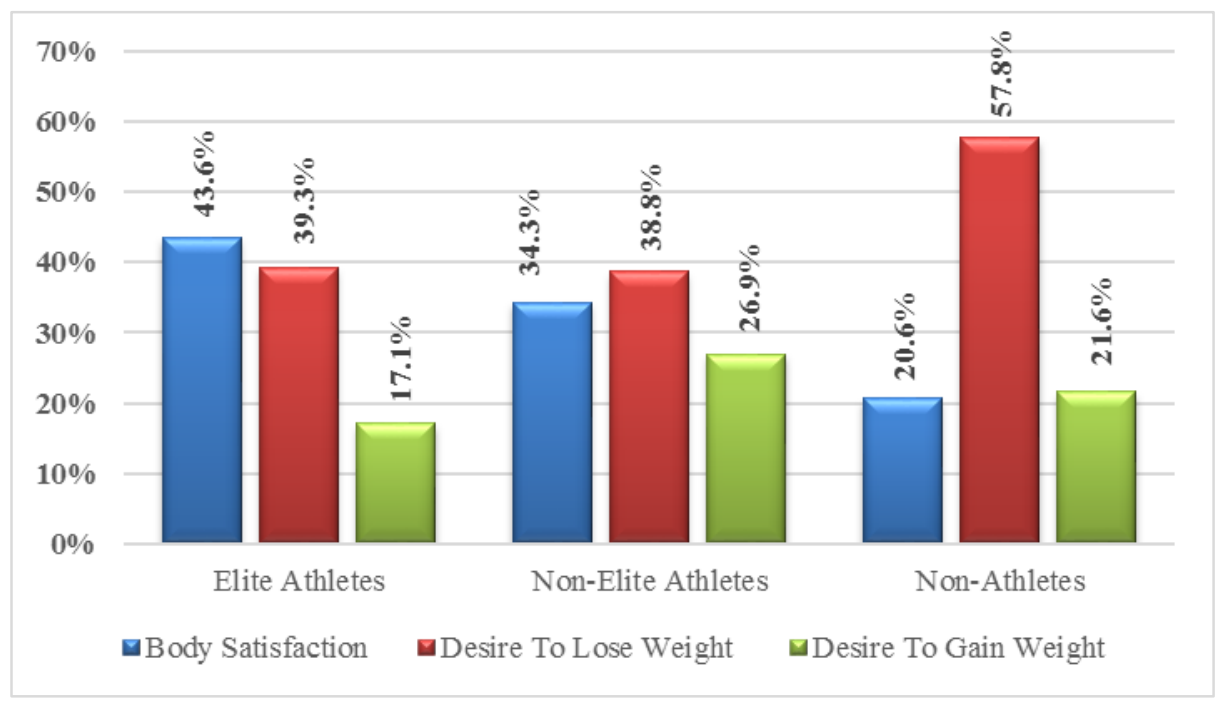

Figure 1: Relative frequency of body dissatisfaction in three groups.

Figure 1 shows $79.3 \%$ of non-athletes (57.8\% desire to lose weight and $21.6 \%$ desire to gain weight), $65.6 \%$ of non-elite athletes (38.8\% desire to lose weight and $26.9 \%$ desire to gain weight), and $56.4 \%$ of elite athletes (39.3\% desire to lose weight and $17.1 \%$ desire to gain weight) are dissatisfied with their body shape.

Also based on figure 4, 58.7\% of elite athletes, $59.1 \%$ of non-elite athletes and $77.6 \%$ non-athletes perceived pressure from family and friends to change weight. Using chi-squire test showed that there is a statistically significant difference in social pressure to change weight among three groups. 


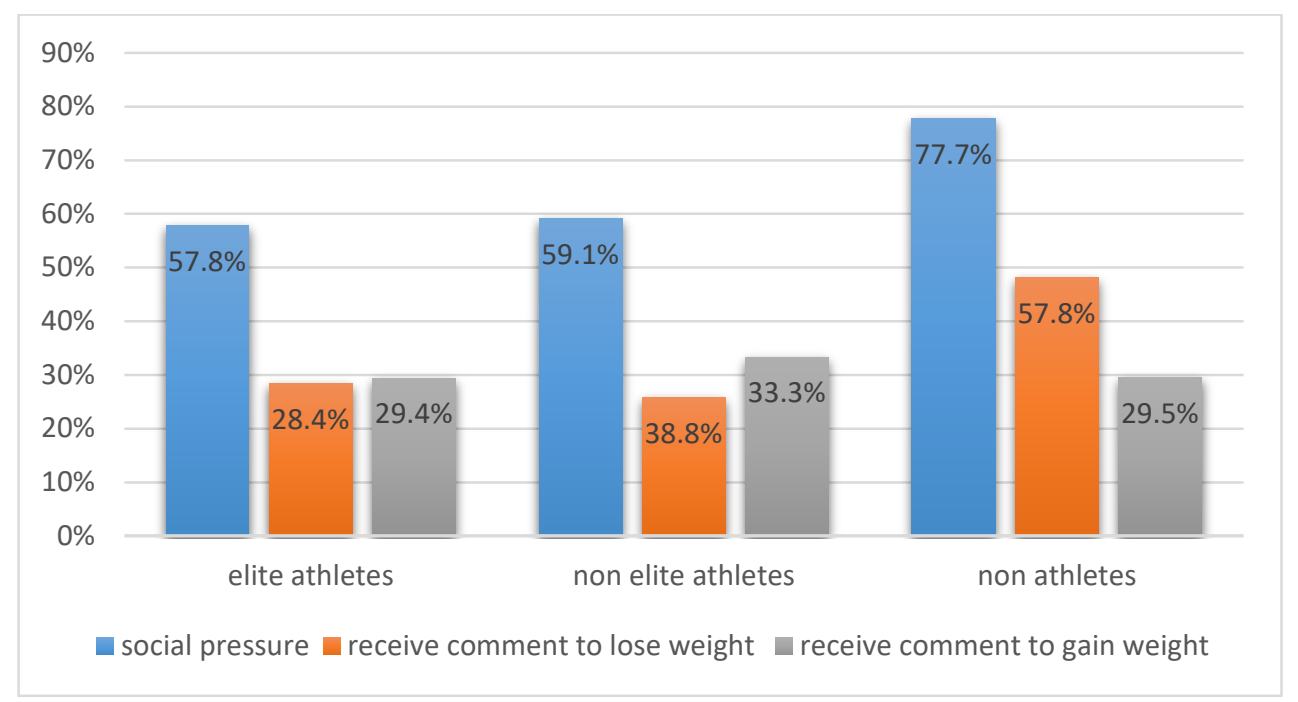

Figure 4: Social Pressure among three groups from family and friends to change weight.

The mean total body mass index of the elite and non-elite athletes is not statistically significant (Table 1). However, BMI categories for all the groups in figure 2 shows the elite athletes, in spite of being the youngest with more physical activity than the other groups, $10.4 \%$ and $1.4 \%$ were overweight and obese respectively. As previous research has pointed out [34, 35, 10], BMI is not a perfect measurement for elite athletes. For example, an elite athlete may have a BMI of 29 or more, irrespective of being very muscular and lean. So it would be imprecise and misleading to consider this athlete as overweight or obese. Also, it should be mentioned that the elite athletes in this study engaged in non-lean sports $[34,35,10]$. 


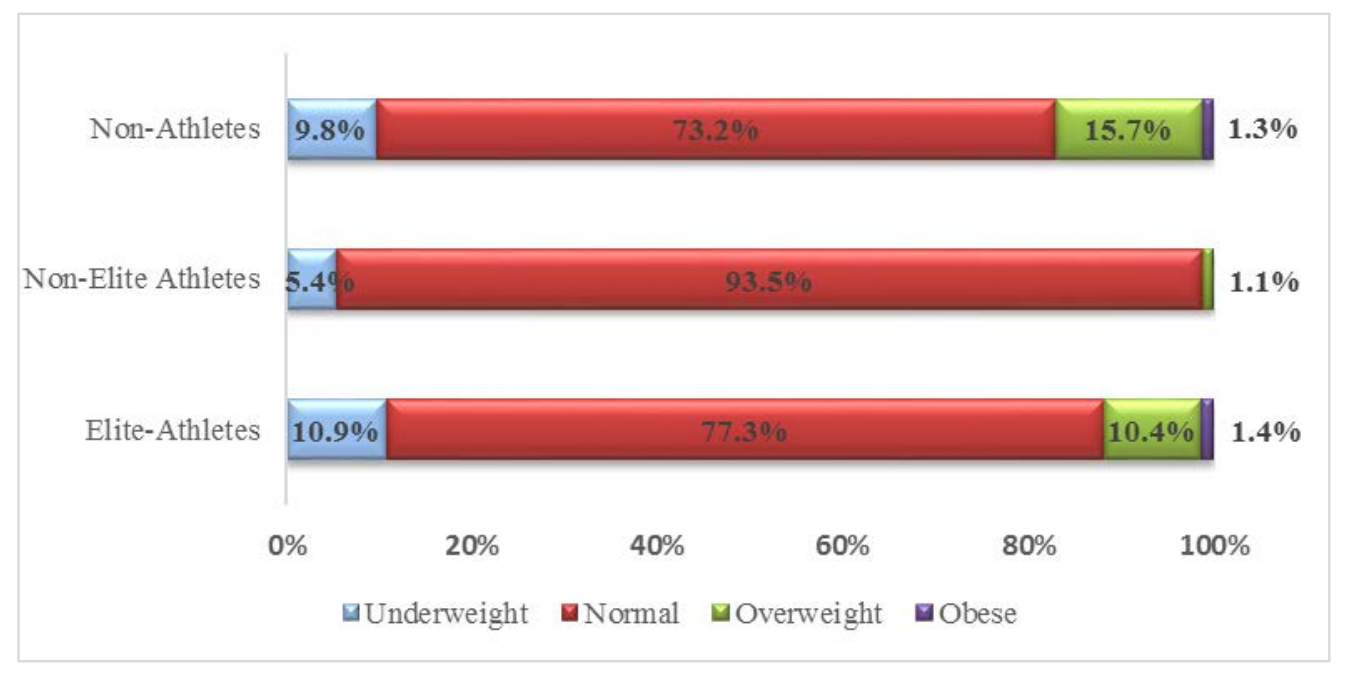

Figure 2: BMI category in three groups.

Table 2 \& 3 display Pearson’s correlations for elite athletes (lower diagonal) and non-elite athletes (upper diagonal) and table 3 for non-elite athletes. As can be seen overall, there was a relatively high correlation between BMI, and social pressure with body dissatisfaction in three groups. The direction of this correlation indicated that body dissatisfaction was positively associated with a higher BMI, and a higher level of social pressure. Moreover, there is a moderate correlation between BMI and body dissatisfaction with weight loss behaviors among three groups.

The result of relative frequency of weight loss behaviors have shown in figure 3. Based on the observation, totally 309 out of 711 participants experience at least one of the weight loss methods in the last two months and $51.1 \%$ of non-athletes experienced potentially unsafe methods. 


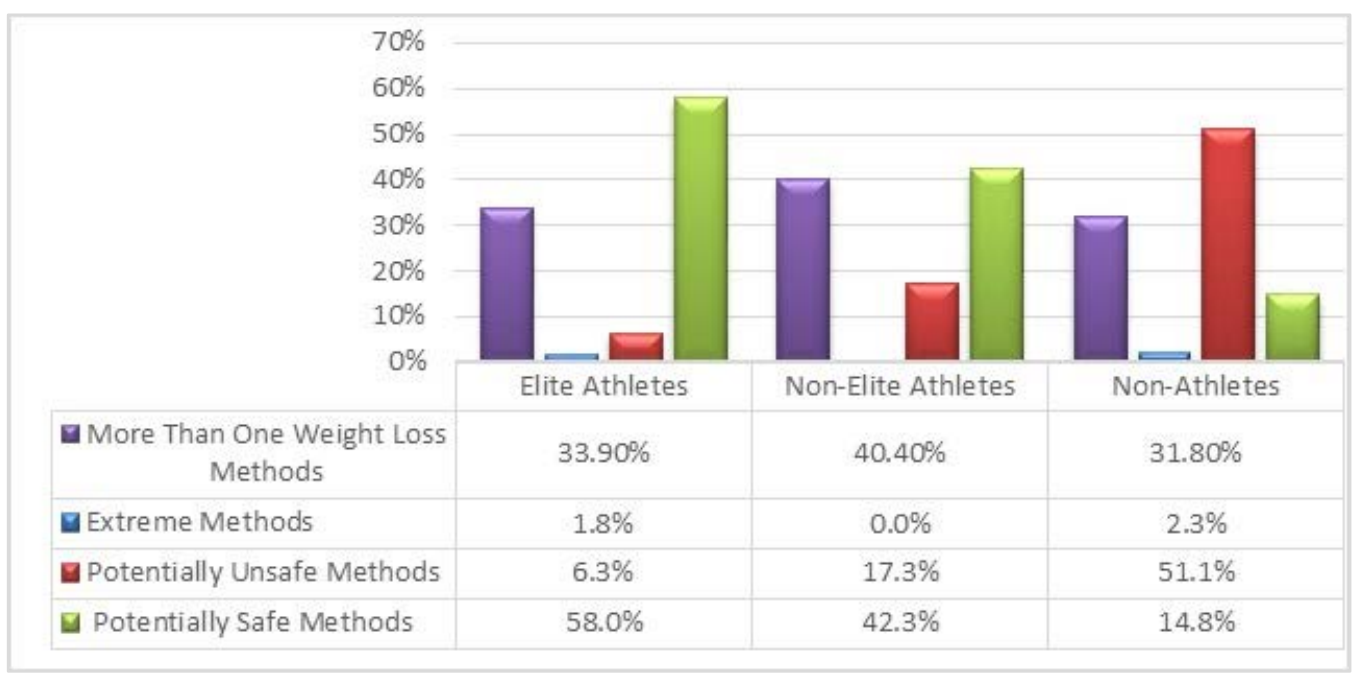

Figure 3: Frequency of weight loss methods among three groups. These frequencies are related to 402 participants who used one of these methods included 96 or $45.5 \%$ of elite athletes, 40 or $43 \%$ of non-elite athletes, and 266 or $65.3 \%$ of non-athlete.

A linear regression (enter method) was conducted for each group to investigate whether any of the significant variables (BMI, current and lifetime sporting activity, social pressure) would predict body dissatisfaction. In each group the final regression model; in elite athletes $\mathrm{F}(2,208)=177.2, \mathrm{p}<0.001$, Adj. R2 $=0.62$, in non-elite athletes $\mathrm{F}(2,90)=233.6$, $\mathrm{p}<0.001$, Adj. R2 =0.83, in non-athletes F $(2,404)=770.9, \mathrm{p}<0.001$, Adj. $\mathrm{R} 2=0.79$, was significant. Social pressure was the strongest predictor of body dissatisfaction in elite athletes $(\beta=0.61, \mathrm{t}=11.70, \mathrm{p}<0.001)$, in non-athletes $(\beta=0.83, \mathrm{t}=16.28, \mathrm{p}<0.001)$, and in non- elite athletes $(\beta=0.83, \mathrm{t}=28.32, \mathrm{p}<0.001)$, followed by BMI in elite athletes $(\beta=$ 0.25, $\mathrm{t}=4.78, \mathrm{p}<0.001)$, in non-elite athletes $(\beta=0.14, \mathrm{t}=2.77, \mathrm{p}<0.001)$ and in nonathletes $(\beta=0.08, \mathrm{t}=2.99, \mathrm{p}<0.001)$. Lifetime sporting activity only in elite athlete was predictor for body dissatisfaction $(\beta=-0.12, \mathrm{t}=2.47, \mathrm{p}<0.05)$. 


\begin{tabular}{|c|c|c|c|c|c|c|c|c|}
\hline & & 1 & 2 & 3 & 4 & 5 & 6 & 7 \\
\hline 1 & Body mass index & & $0.60^{* *}$ & $0.55^{* *}$ & $0.34^{* *}$ & -0.11 & 0.02 & -0.04 \\
\hline 2 & Body dissatisfaction & $0.62^{* *}$ & & $0.90^{* *}$ & $0.47^{* *}$ & -0.10 & 0.08 & -0.12 \\
\hline 3 & Social pressure & $0.59^{* *}$ & $0.76^{* *}$ & & $0.38^{* *}$ & 0.08 & 0.04 & -0.10 \\
\hline 4 & Weight loss behaviors & $0.32^{* *}$ & $0.38^{* *}$ & $0.29^{* *}$ & & 0.01 & 0.10 & -0.17 \\
\hline 5 & Life time sporting activity & -0.21 & $-0.26^{* *}$ & $-0.12^{* *}$ & $0.19^{* *}$ & & 0.19 & $0.74^{* *}$ \\
\hline 6 & Day per week & $-0.16^{*}$ & $-0.22^{* *}$ & $-0.16^{*}$ & -.012 & $0.51^{* *}$ & & $0.47^{* *}$ \\
\hline 7 & Hours per session & -0.05 & -0.07 & -0.06 & -0.03 & $0.27^{* *}$ & $0.54^{* *}$ & \\
\hline
\end{tabular}

Table 2: Pearson's correlations between variables among elite and non-elite athletes. Values for female elite athletes lie below the diagonal; values for non-elite athletes lie above the diagonal. ${ }^{*} \mathrm{p}<.05,{ }^{* *} \mathrm{p}<.01$.

\begin{tabular}{|c|l|l|l|l|l|l|l|l|}
\hline & & 1 & 2 & 3 & 4 & 5 & 6 & 7 \\
\hline 1 & Body mass index & & & & & & & \\
\hline 2 & Body dissatisfaction & $0.66^{* *}$ & & & & & & \\
\hline 3 & Social pressure & $0.68^{* *}$ & $0.88^{* *}$ & & & & & \\
\hline 4 & Weight loss behaviors & $0.37^{* *}$ & $0.44^{* *}$ & $0.40^{* *}$ & & & & \\
\hline 5 & Life time physical activity & 0.07 & 0.06 & 0.03 & -0.02 & & & \\
\hline 6 & Days per week & 0.14 & 0.15 & 0.18 & -0.14 & $0.32^{* *}$ & & \\
\hline 7 & Hours per session & -0.11 & -0.04 & 0.08 & -0.18 & 0.08 & -0.08 & \\
\hline
\end{tabular}

Table 3: Pearson's correlations between variables among non-athletes $* \mathrm{p}<.05,{ }^{* *} \mathrm{p}<.01$

\section{Discussion}

The findings of the current study show that athletes have a lower level of body dissatisfaction (table1) than non-athletes which is parallel with results of Smolak et al.; Hausenblas et al. \& Martinsen et al [36, 34, 17]. Our results propose that female elite athletes do not have a different level of body dissatisfaction and social pressure from nonelite athletes (table1). It might be originated from the sports major because elite athletes in this report competed in non-lean sports such as rugby, canoe polo, track and field, 
basketball, and volleyball that emphasize on physical strength more than endurance. Although elite athletes are believed to face extra social pressure to achieve an ideal body shape [37], this study shows that non-athlete perceive higher social pressure than their elite and non-elite counterparts (figure1). Interestingly, this study illustrates that $29.4 \%$ of female elite athletes perceive social pressure for weight gain (figure 4). It can be based on the fact that either $10.9 \%$ of them were underweight which persuades them to gain weight, or it may result from silent pressure to gain muscle as suggested by Krane et al., that female athletes, depending on their sports major, may desire a more muscular body [38].

In this study, all female athletes had lower degrees of body dissatisfaction and social pressure than non-athletes for several reasons. First, possible mechanisms explaining improved body satisfaction in athletes could be related to the level of physical activity, especially in non-lean sports. This factor can enhance muscular strength and decreased total skinfold thickness, so it would improve athlete body shape, self-esteem, physical selfworth, and body satisfaction because athletes may observe major differences between them and non-athletes in body shape [15]. It is suggested that physical activity is a potential therapy for body dissatisfaction among women [39]. In the above study physical activity refers to weight training. However, an athlete typically does weight training during the competitive season and off-season to increase size, speed, and endurance [40]. Second, female athletes may want to have an ideal body shape that is related to the sports major. For some of them muscularity and strength may be their ideal sports body shape [40]. This condition seems to be met by the participants of this study too because most of them competed in sports majors which lean body shape was not considerable. On the other hand, due to the high level of physical activities, athletes may resemble an aesthetic ideal for 
body shape [34]. Correspondingly, in current study athletes in both groups had a significantly lower BMI than non-athletes (Table1). Since BMI is an effective factor on body dissatisfaction [10, 41], is logical that athletes with lower BMI in compared with nonathletes have a higher body satisfaction. Also, the high correlations between BMI with body dissatisfaction and social pressure in three groups (table2) confirm that why athletes are more satisfied with their body shape than non-athletes. When body shape does not match the beauty standards of society, people may continuously face with weight-related teasing from peers and family which may result in low self-esteem, low self-worth, and body dissatisfaction [42].

It should be noted that body dissatisfaction can induce the person to use weight loss methods to gratify the people around them [5]. In this study, $48.2 \%$ of non-athletes perceived social pressure to lose weight, while merely $17 \%$ of them were either overweight or obese and nearly half of the participants with normal BMI perceive social pressure too. Since no other study has been carried out among Iranian female athletes, is not possible to compare our findings with Iranian female athletes. Nevertheless, the prevalence of body dissatisfaction among non-athletes is comparable among Iranian women. For example, Abdollahi et al., Nobakht et al., showed that body dissatisfaction is common among women in Iran [43, 44]. However, Alipoor et al. by employing BSQ to assess body dissatisfaction, estimated 92.9\% of female students in the south of Iran were satisfied with their body shape [45]. This inconsistency with above study is because, the average BMI of non-athletes in our result, was higher $(\mathrm{m}=21.07)$.

In consist with other studies $[46,47,10,15,48]$ perceived pressure from family and friends to change weight is a significant predictor of body dissatisfaction. Another predictor 
for body dissatisfaction is BMI among three groups, which is in line with the previous studies too [10, 15, 48]. Moreover, lifetime sporting activity can be another predictor of body dissatisfaction among elite athletes $(\beta=-0.12)$. Although it is not a sufficiently strong predictor, it is necessary to report this result because it can highlight the relative significance of lifetime sporting activity to predict some portion of body dissatisfaction among elite athletes.

Weight loss methods seem to be different among the three groups. In other words, common weight loss methods were used by elite and non-elite athletes were potentially safe methods, while unsafe methods were common among non-athlete group. Non-elite athletes did not use extreme methods because most of the non-elite athletes were physical education students and they were likely to be aware of the side effect of extreme methods on their health and their performance. In contrast, 58\% of elite athletes used potentially safe method (excessive exercise). It can be explained based on the fact that, although excessive exercise is known as diagnostic criteria for anorexia or bulimia nervosa [49], it may account for a factor in successful athletic performance. In this line, for some athletes, excessive exercise and weight control behaviors may reflect their commitment to the sports carrier rather than psychological disorder such as body dissatisfaction [37]. It is suggested that although there is a similarity between characteristics of "good athletes" and traits of anorectic patients, the excessive exercise in athletes is likely to be neither pathological nor related to anorectic and bulimic behaviors [49]. It is alarming that $45.5 \%$ of elite athletes, $43 \%$ of non-elite athletes, and $65.3 \%$ of non-athletes used weight loss methods, while the majority of them were classified within a normal range of BMI (see fig2). In correspondence with these results, Litchy reported $85 \%$ of non-overweight girls were 
engaged in weight control behaviors, and therefore, most of the non-athletes do not regard exercise as a way to achieve their ideal body shape [2]. As a consequence, the majority of them use pathogenic weight loss behaviors to obtain this purpose. As an example, our findings indicate that merely 60 (14.8\%) of all non-athlete participants did exercise, but not as a regular activity, albeit as a method of losing weight. Irrespective of the fact that few female elite athletes and non-athletes utilized extreme methods to lose weight, it should severely be noted that these methods have undesirable consequences, such as reduce sports performance in elite athletes or injurious effects on health in both groups [17].

There is a direct relationship between body dissatisfaction and social pressure with weight loss behaviors [20] that our results show that this direct relationship is moderate in strength and statistically significant among all the three groups. Our findings highlight the importance of body dissatisfaction and social pressure on weight loss behaviors.

Finally, although non-Western societies are said to value plumpness rather than thinness [21], the results of the current study indicate that in the whole samples, $81.9 \%$ of participants selected an underweight figure (1 to 4 of PFRS) as their desired body shape. Exposure to Western media might be a contributing factor to a high prevalence of body dissatisfaction and social pressure to change weight among Iranian women. Although the use of non-native television and Western media are formally banned in Iran since 1978, researchers have shown that recently, non-native television in urban regions in Iran is widespread [50]. Rasmanesh et al. illustrated that over the past decades, access to worldwide media such as the Internet and TV causes Western culture gradually influence women in Iran to change their beauty standards [51]. To support the fact, Rahimi reported that Iran is the second country that has the most internet users in the Middle-East [52]. 


\section{Conclusion}

Our findings demonstrate that variables such as body dissatisfaction, social pressure, and pathogenic weight loss behaviors are more pervasive among non-athlete women than athletes. The results show that there is a negative correlation between body dissatisfaction and lifetime physical activity. Therefore, we suggest that physical activity is not only a risk factor for body dissatisfaction, but also can be regarded as a preventive strategy. As the results show, an exclusively high level of physical activity may not account for body dissatisfaction, social pressure, and pathogenic weight loss behaviors; but other contributory factors such as perfectionism, self-esteem, psychological characteristic, and sports major are probably more effective. It should be noted that body dissatisfaction in athletes may be more related to psychological elements than sports environmental factors. Overall the results of the present study demonstrated that among Iranian women body dissatisfaction, social pressure, and weight loss behaviors are as common as Western society.

As previous researchers had recommended, in our study following concepts were taken into account: First, the study examined a large representative sample of female elite athletes and compared them with the matched control groups (non-elite athletes and non-athletes). Second, in order to reduce the predictive error of self-reported BMI [14] especially among overweight people [53], variables were measured by standard instruments. Third, the number of training days/hours per week was calculated to analyze the relationship between physical activity and body dissatisfaction [10]. 
Further researches are needed to illustrate the related factors that are perhaps likely to be associated with body dissatisfaction in female athletes and non-athletes such as media influence, self-esteem, and perfectionism.

\title{
CONFLICT OF INTEREST
}

The authors report no conflicts of interest in this work.

\author{
AUTHOR'S CONTRIBUTIONS \\ Donya Nemati - Substantial contributions to conception and design, Acquisition of data, analysis and \\ interpretation of data, Drafting the article, revising it critically for important intellectual content, Final \\ approval of the version to be published. All participants were interviewed by Donya Nemati to collect \\ unbiased data. \\ Rahimi-nia - Analysis and interpretation of data, revising it critically for important intellectual content, \\ Final approval of the version to be published \\ Mohebi - Final approval of the version to be published
}

\section{References}

[1] F. P. d. OliveiraI, M. L. M. BosiII, P. d. S. VigárioIII and R. d. S. VieiraIII, "Eating behavior and body image in athletes," Revista Brasileira de Medicina do Esporte, vol. 9, pp. 348-356, 2003.

[2] J. M. Liechty, "Body image distortion and three types of weight loss behaviors among nonoverweight girls in the United States," Journal of Adolescent Health, vol. 47, pp. 176-182, 2010.

[3] E. Smeets, A. Jansen, E. Vossen, L. Ruf and A. Roefs, "Feeling body dissatisfied after viewing thinideal pictures is mediated by self-activation," Body image, vol. 7, pp. 335-340, 2010. 
[4] T. Jackson and H. Chen, "Sociocultural predictors of physical appearance concerns among adolescent girls and young women from China," Sex Roles, vol. 58, pp. 402-411, 2008.

[5] R. N. Ata, A. B. Ludden and M. M. Lally, "The effects of gender and family, friend, and media influences on eating behaviors and body image during adolescence," Journal of Youth and Adolescence, vol. 36, pp. 1024--1037, 2007.

[6] R. F. Rodgers, S. J. Paxton and H. Chabrol, "Effects of parental comments on body dissatisfaction and eating disturbance in young adults: A sociocultural model," Body image, vol. 6, pp. 171-177, 2009.

[7] H. Keery, K. Boutelle, P. van den Berg and J. K. Thompson, "The impact of appearance-related teasing by family members," Journal of Adolescent Health, vol. 37, pp. 120-127, 2004.

[8] V. a. S. A. R. a. T. J. K. Phares, "Gender differences in peer and parental influences: Body image disturbance, self-worth, and psychological functioning in preadolescent children," Journal of Youth and Adolescence, vol. 33, pp. 421-429, 2004.

[9] L. A. Ricciardelli and M. P. McCabe, "Sociocultural and individual influences on muscle gain and weight loss strategies among adolescent boys and girls," Psychology in the Schools, vol. 40, pp. 209224, 2003.

[10] V. Swami, L. Steadman and M. J. Tovee, "A comparison of body size ideals, body dissatisfaction, and media influence between female track athletes, martial artists, and non-athletes," Psychology of Sport and Exercise, vol. 10, pp. 609-614, 2009.

[11] J. Reed and D. S. Ones, "The effect of acute aerobic exercise on positive activated affect: A metaanalysis," Psychology of Sport and Exercise, vol. 7, pp. 477-514, 2006.

[12] M. L. LePage and J. H. Crowther, "The effects of exercise on body satisfaction and affect," Body image, vol. 7, pp. 124-130, 2010.

[13] T. M. Dunkel, D. Davidson and S. Qurashi, "Body satisfaction and pressure to be thin in younger and older Muslim and non-Muslim women: The role of Western and non-Western dress preferences," Body Image, vol. 7, pp. 56-65, 2010. 
[14] J. L. Engstrom, S. A. Paterson, A. Doherty, M. Trabulsi and K. L. Speer, "Accuracy of self-reported height and weight in women: an integrative review of the literature," Journal of Midwifery and Women’s Health, vol. 48, pp. 338-345, 2003.

[15] H. a. D. D. S. Hausenblas, D. Fleming and D. Connaughton, "Body image in middle school children," Eating and Weight Disorders-Studies on Anorexia, Bulimia and Obesity, vol. 7, pp. 244-248, 2002.

[16] V. Costarelli and D. Stamou, "Emotional intelligence, body image and disordered eating attitudes in combat sport athletes," Journal of Exercise Science and Fitness, vol. 7, pp. 104--111, 2009.

[17] M. Martinsen, S. Bratland-Sanda, A. K. Eriksson and J. Sundgot-Borgen, "Dieting to win or to be thin? A study of dieting and disordered eating among adolescent elite athletes and non-athlete controls," British Journal of Sports Medicine, vol. 44, pp. 70-76, 2010.

[18] M. A. Kennedy, L. Templeton, A. Gandhi and B. B. Gorzalka, "Asian body image satisfaction: Ethnic and gender differences across Chinese, Indo-Asian, and European-descent students," Eating Disorders, vol. 12, pp. 321-336, 2004.

[19] D. a. M. M. Mellor, L. Ricciardelli, J. Yeow, N. Daliza and others, "Sociocultural influences on body dissatisfaction and body change behaviors among Malaysian adolescents," Body Image, vol. 6, pp. 121-128, 2009.

[20] X. Xu, D. Mellor, M. Kiehne, L. A. Ricciardelli, M. P. McCabe and Y. Xu, "Body dissatisfaction, engagement in body change behaviors and sociocultural influences on body image among Chinese adolescents," Body image, vol. 7, pp. 156-164, 2010.

[21] N. L. Soh, S. W. Touyz and L. J. Surgenor, "Eating and body image disturbances across cultures: A review," European Eating Disorders Review, vol. 14, pp. 54-65, 2006.

[22] H. Dadgostar, M. Razi, A. Aleyasin, T. Alenabi and S. Dahaghin, "The relation between athletic sports and prevalence of amenorrhea and oligomenorrhea in Iranian female athletes," BMC Sports Science, Medicine and Rehabilitation, vol. 1, p. 1, 2009.

[23] M. F. Reinking and L. E. Alexander, "Prevalence of disordered-eating behaviors in undergraduate female collegiate athletes and nonathletes," Journal of athletic training\}, vol. 40, p. 47, 2005. 
[24] J. Sundgot-Borgen and S. Larsen, "Pathogenic weight-control methods and self-reported eating disorders in female elite athletes and controls," Scandinavian journal of medicine and science in sports, vol. 3, pp. 150-155, 1993.

[25] V. Swami, N. Salem, A. Furnham and M. Tove, "Initial examination of the validity and reliability of the female Photographic Figure Rating Scale for body image assessment," Personality and Individual Differences, vol. 44, pp. 1752-1761, 2008.

[26] L. A. Ricciardelli, M. P. McCabe, J. Lillis and K. Thomas, "A longitudinal investigation of the development of weight and muscle concerns among preadolescent boys," Journal of Youth and Adolescence, vol. 35, pp. 168-178, 2006.

[27] E. Stice and S. K. Bearman, "Body-image and eating disturbances prospectively predict increases in depressive symptoms in adolescent girls: a growth curve analysis," Developmental psychology, vol. 37, p. 597, 2001.

[28] M. P. McCabe and L. A. Ricciardelli, "A prospective study of pressures from parents, peers, and the media on extreme weight change behaviors among adolescent boys and girls," Behaviour research and therapy, vol. 43, pp. 653-668, 2005.

[29] M. Janghorbani, M. a. W. W. C. Amini, M. M. Gouya, A. Delavari, S. Alikhani and A. Mahdavi, "First nationwide survey of prevalence of overweight, underweight, and abdominal obesity in Iranian adults," Obesity, vol. 15, pp. 2797-2808, 2007.

[30] H. R. Khalkhali, "High prevalence of overweight and obesity in women of Islamshahr, Iran," Asia Pac J Clin Nutr, vol. 14, pp. 169-172, 2005.

[31] S. B. Green and N. J. Salkind, "Using SPSS for Windows and Macintosh: Analyzing and understanding data," Prentice Hall Press, 2010.

[32] A. Gelman, "Analysis of variance-why it is more important than ever," The Annals of Statistics, vol. 33, pp. 1-53, 2005.

[33] P. Sedgwick, "Pearson’s correlation coefficient," BMJ, vol. 345, p. e4483, 2012. 
[34] H. A. Hausenblas and D. S. Downs, "Comparison of body image between athletes and nonathletes: A meta-analytic review," Journal of Applied Sport Psychology, vol. 13, pp. 323-339, 2001.

[35] M. P. Olmsted and T. McFarlane, "Body weight and body image," BMC women's health, vol. 4, p. 1, 2004.

[36] L. Smolak, S. K. Murnen, A. E. Ruble and others, "Female athletes and eating problems: a metaanalysis," International journal of eating disorders, vol. 27, pp. 371-380, 2000.

[37] S. Byrne and N. McLean, "Elite athletes: effects of the pressure to be thin," Journal of Science and Medicine in Sport, vol. 5, pp. 80--94, 2002.

[38] V. Krane, P. Y. Choi, S. M. Baird, C. M. Aimar and K. J. Kauer, "Living the paradox: Female athletes negotiate femininity and muscularity," Sex roles, vol. 50, pp. 315-329, 2004.

[39] E. DEPCIK and L. WILLIAMS, "Weight training and body satisfaction of body-image-disturbed college women," Journal of Applied Sport Psychology, vol. 16, pp. 287--299, 2004.

[40] A. Smith and T. Petrie, "Reducing the risk of disordered eating among female athletes: A test of alternative interventions," Journal of Applied Sport Psychology, vol. 20, pp. 392-407, 2008.

[41] W. C. Lynch, D. P. a. W. E. Heil and M. D. Havens, "Body dissatisfaction mediates the association between body mass index and risky weight control behaviors among White and Native American adolescent girls," Appetite, vol. 51, pp. 210-213, 2008.

[42] J. E. Menzel, L. M. Schaefer, N. L. Burke, L. L. Mayhew, M. T. Brannick and J. K. Thompson, "Appearance-related teasing, body dissatisfaction, and disordered eating: A meta-analysis," Body image, vol. 7, pp. 261-270, 2010.

[43] P. a. M. T. Abdollahi, "Eating disorder symptoms and body image concerns in Iran: comparisons between Iranian women in Iran and in America," International Journal of Eating Disorders, pp. 259268, 2001.

[44] M. Nobakht and M. Dezhkam, "An epidemiological study of eating disorders in Iran," International Journal of Eating Disorders, vol. 28, pp. 265-271, 2000. 
[45] S. Alipoor, A. Goodarzi, M. Nezhad and L. Zaheri, "Analysis of the relationship between physical self-concept and body image dissatisfaction in female students," Journal of Social Sciences, vol. 5, pp. 60-66, 2009.

[46] A. E. Field, C. A. Camargo, C. B. a. B. C. S. Taylor, S. B. Roberts and G. A. Colditz, "Peer, parent, and media influences on the development of weight concerns and frequent dieting among preadolescent and adolescent girls and boys," Pediatrics, vol. 107, pp. 54-60, 2001.

[47] E. Stice and K. Whitenton, "Risk factors for body dissatisfaction in adolescent girls: a longitudinal investigation," Developmental psychology, vol. 38, p. 669, 2002.

[48] E. R. Sands and J. Wardle, "Internalization of ideal body shapes in 9--12-year-old girls," International Journal of Eating Disorders, vol. 33, pp. 193-204, 2003.

[49] R. A. Thompson and R. Trattner Sherman, "Athletes, athletic performance, and eating disorders: Healthier alternatives," Journal of Social Issues, vol. 55, pp. 317-337, 1999.

[50] M. Soltanifar and N. Malekian, "Analysis of the MBC Persian Satellite Network Programs in order to Achieve Communicational Culture and Techniques used in Transmitting Messages," European Journal of Social Sciences, vol. 17, p. 471, 2010.

[51] R. Rastmanesh, M. E. Gluck and Z. Shadman, "Comparison of body dissatisfaction and cosmetic rhinoplasty with levels of veil practicing in Islamic women," International Journal of Eating Disorders, vol. 42, pp. 339-345, 2009.

[52] B. Rahimi, "Cyberdissent: the Internet in revolutionary Iran," Middle East, vol. 7, p. 102, 2003.

[53] X. Zhou, M. J. Dibley, Y. Cheng, X. Ouyang and H. Yan, "Validity of self-reported weight, height and resultant body mass index in Chinese adolescents and factors associated with errors in selfreports," BMC public health, vol. 10, 2010. 